\title{
Coreless Vortices in Rotating Two-Component Quantum Droplets
}

\author{
H. Saarikoski, ${ }^{1}$ A. Harju,${ }^{2}$ J. Christensson, ${ }^{1}$ S. Bargi,${ }^{1}$ M. Manninen,${ }^{3}$ and S. M. Reimann ${ }^{1, *}$ \\ ${ }^{1}$ Mathematical Physics, Lund Institute of Technology, SE-22100 Lund, Sweden \\ ${ }^{2}$ Helsinki Institute of Physics and Department of Applied Physics, \\ Helsinki University of Technology, Espoo, Finland \\ ${ }^{3}$ Nanoscience Center, Department of Physics, FIN-40014, University of Jyväskylä, Jyväskylä, Finland
}

\begin{abstract}
The rotation of a quantum liquid induces vortices to carry angular momentum. When the system is composed of multiple components that are distinguishable from each other, vortex cores in one component may be filled by particles of the other component, and coreless vortices form. Based on evidence from computational methods, here we show that the formation of coreless vortices occurs very similarly for repulsively interacting bosons and fermions, largely independent of the form of the particle interactions. We further address the connection to the Halperin wave functions of non-polarized quantum Hall states.
\end{abstract}

PACS numbers: 67.10.-j 03.75.Lm 05.30.-d 73.21.La

Setting a trapped Bose-Einstein condensate (BEC) rotating, as for example by stirring the bosonic droplet with lasers [1], quantum-mechanical "twisters" may form in the cloud to carry angular momentum. Such vortices consist of a hole in the particle density, associated with a quantized phase change in the order parameter around the core. With increasing rotation the vortices enter the bosonic cloud from its periphery [2, 3] one by one, finally leading to the well-known vortex lattice when angular momentum is high. The first experiment on vortices in a BEC [4] was in fact done with a two-component gas, as suggested by Williams and Holland [5]. Atoms of ${ }^{87}$ Rubidium were trapped in two distinguishable hyperfine spin states, and vortices could be created in one component while the other component was at rest. At high rotation, the vortex lattices of the two components are interlaced and 'coreless' vortices form with one component filling the vortex cores of the other component as a consequence of their mutual repulsion $[6]$. This was also experimentally observed by Schweikhard et al. [7].

While usually attributed to superfluidity, quantized vortices as they occur in condensates of bosonic atoms may even appear with trapped rotating fermion systems [8, 9, 10]. Vortex patterns in few-electron quantum dots at strong magnetic fields appear as hole-like quasiparticles in much analogy to the bosonic case [10, 11]. Moreover, there often exists a simple transformation of the fermionic wave function into the bosonic one [12] and a close theoretical connection between rotating bosonic systems and quantum Hall states 13. Depending on the angular momentum vortices may either nucleate to a vortex cluster, just like in the bosonic case [2, 4, 14], or appear as particle-vortex composites in the finite-size precursors of polarized [8, 9, 10, 11, 15] and unpolarized [16, 17] fractional quantum Hall states. In experiments rotating two-component fermion droplets may be realized with trapped dipolar fermion gases [18], or with electrons in quasi two-dimensional quantum dots [19], where the two components are distinguished by the spin quantization axis of the electrons, and coreless vortices are expected to form in the regime of rapid rotation.

In this Letter, we report numerical evidence for the remarkable similarity of coreless vortices in rotating twocomponent quantum droplets regardless of the particle statistics and the form of particle interactions.

We focus on rapid rotation, and restrict our analysis to a two-dimensional harmonic trapping potential of oscillator frequency $\omega$. For a droplet composed of two distinguishable species $\mathrm{A}$ and $\mathrm{B}$ with particle number $N=N_{\mathrm{A}}+N_{\mathrm{B}}$, the Hamiltonian is

$\hat{H}=\sum_{i=1}^{N_{\mathrm{A}}+N_{\mathrm{B}}}\left(\frac{\mathbf{p}_{i}^{2}}{2 m}+\frac{1}{2} m \omega^{2} \mathbf{r}_{i}^{2}\right)+\frac{1}{2} \sum_{i \neq j=1}^{N_{\mathrm{A}}+N_{\mathrm{B}}} V\left(\mathbf{r}_{i}, \mathbf{r}_{j}\right)-\Omega L$,

where $\mathbf{r}=(x, y), m$ is the mass (equal for both species), $\Omega$ is the frequency of the external rotation, and $L$ is the (z-component) of angular momentum. $V$ is the particle-particle interaction potential, which is Coulombic, $V=e^{2} /\left(4 \pi \epsilon\left|\mathbf{r}_{i}-\mathbf{r}_{j}\right|\right)$, in the case of electrons in a quantum dot. For bosonic condensates we have used both the Coulombic potential and the contact potential $V=U \delta\left(\mathbf{r}_{i}-\mathbf{r}_{j}\right)$, where $U=4 \pi \hbar^{2} a / m$ and $a$ is the scattering length (here assumed to be the same for both components), which is more realistic for cold atom condensates. In semiconductor quantum dots, the Zeeman splitting can be engineered to be zero, as for example in GaAs $/ \mathrm{Al}_{x} \mathrm{Ga}_{1-x} \mathrm{As}$-based systems [20, 21]. In this paper we explicitly assume that the Zeeman splitting is negligible which leads to symmetry between the two spin components.

The eigenstates of the Hamiltonian Eq. (11) are calculated by numerical diagonalization in the lowest Landau Level (LLL) (for details see Refs. [10] and [22]). For large systems, however, we applied the spin-density-functional method as described in Ref. [8].

Pair-correlation functions are commonly used to analyze the structure of the many-body wave function but integrations average out the effect of phase singularities 
a)

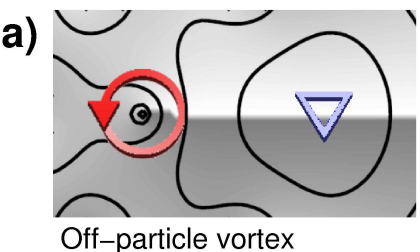

c)

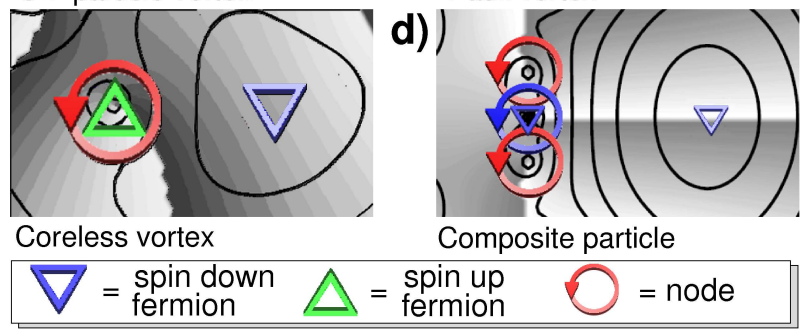

FIG. 1: (Color online): Identification of vortex structures in a restricted wave function. The figures show RWFs for spin- $1 / 2$ fermions. The triangles show the fixed particle positions and the probing particle is marked with light blue. Contours show the magnitude of the RWF (on a logarithmic scale), and the gray-scale shows the phase (darkest gray $=0$, lightest gray $=$ $2 \pi)$. a) Vortex which is not attached to any particle. b) Pauli vortex (blue) which is mandated by the wave function antisymmetry between interchange of indistinguishable fermions. c) Coreless vortex where a vortex core in one component is filled by a particle in the other component. d) A particlevortex composite made up of a fermion (with a Pauli vortex) and two additional nodes which are bound to the particle.

which are signatures of vortices. Therefore, we investigate the nodal structure of the wave function using restricted (conditional) wave functions (RWF) [8] which essentially depict the structure of the $2 N$-dimensional eigenstate of the Hamiltonian Eq. (1), $\Psi\left(\mathbf{r}_{1}, \ldots, \mathbf{r}_{N}\right)$, in a two-dimensional subspace by fixing all but one probing particle:

$$
\psi_{\mathrm{RWF}}(\mathbf{r})=\frac{\Psi\left(\mathbf{r}, \mathbf{r}_{2}^{*}, \ldots, \mathbf{r}_{N}^{*}\right)}{\Psi\left(\mathbf{r}_{1}^{*}, \mathbf{r}_{2}^{*}, \ldots, \mathbf{r}_{N}^{*}\right)}
$$

Here, the denominator is for normalization and the fixed particle positions $\mathbf{r}_{2}^{*}, \ldots, \mathbf{r}_{N}^{*}$ are determined such that the norm of $\psi_{\mathrm{RWF}}$ is maximized and $\mathbf{r}_{1}^{*}$ is the most probable position of the probing particle. The RWFs are two-dimensional complex functions where vortices can be identified directly as nodes with a phase change of a multiple of $2 \pi$ in a path around them. Figure 1 provides a guide to the identification of vortex structures in RWFs.

In the following, we exclusively discuss equal populations of the two species A and B. We first study coreless vortex structures in a 6-particle system with $N_{\mathrm{A}}=N_{\mathrm{B}}=3$, using the ED method in the LLL. For a single-component fermion system the state with the lowest angular momentum in the LLL is called the maximum-density-droplet (MDD) 23], and has angular momentum $L_{\mathrm{MDD}}=N(N-1) / 2$, which is the minimum angular momentum compatible with the Pauli prin- ciple. The corresponding wave function is $\Psi_{\mathrm{MDD}}=$ $\Pi_{i<j}^{N}\left(z_{i}-z_{j}\right) e^{-\frac{1}{2} \Sigma_{k}\left|z_{k}\right|^{2}}$ (with $z=x+i y$ ), where the zeros in the polynomial are interpreted as Pauli vortices [8].

For single-component systems a simple transformation removes the attachment of Pauli vortices to each fermion and thus leads to a symmetric wave function 24] which usually describes, to a significant degree of accuracy [12], bosonic states at $L_{\mathrm{Boson}}=L_{\text {Fermion }}-L_{\mathrm{MDD}}$. In twocomponent systems Pauli vortices are only attached to particles of the same spin and there exist states without bosonic counterparts at $L_{\text {Fermion }}<L_{\mathrm{MDD}}$. Thus, we consider only the angular momentum regime $L_{\text {Fermion }} \geq$ $L_{\mathrm{MDD}}$.

Figure 2 shows the RWFs of two-component bosonic and fermionic systems for Coulomb interactions, at ground-state angular momenta as given in each panel. Removal of a single vortex from each fermion position (for both components) leaves a wave function whose nodal structure shows formation of an equal number of coreless vortices in both fermionic and bosonic systems. These can be identified in the RWFs as vortices in one component that coincide with probability maxima in the opposite component, in order to minimize the interaction energy. States at intermediate angular momenta show a vortex entry from the droplet periphery, similar to that in single-component systems [15, 25]. Just as in the bosonic case, the two components of a fermion system first separate (Fig. 2b) 22 and, as the angular momentum increases, form interlaced vortex patterns (Fig. 2 $\mathrm{d}$ ). These features in the RWFs are well reflected also in the pair correlations of bosonic systems (Fig. 3). Furthermore, we find that for bosons interacting with a contact interaction the overlaps with the corresponding states with Coulomb interaction are higher than 0.98 which shows that the coreless vortex formation is largely independent of the form of the interaction.

There is a trend for the localization of particles to increase with angular momentum (and thus vorticity), as manifested by a narrowing of the RWF peak around the most probable particle position (Fig. 2). The effect is more clearly seen in fermion systems where the number of vortices is higher.

For larger system sizes, the diagonalization of the many-body Hamiltonian Eq. (10) becomes numerically impossible. In bosonic systems one often turns to mean-field methods such as the Gross-Pitaevskii (GP) equation. In the case of fermions, we can make use of density-functional theory (DFT), as previously described for one-component droplets in Ref. [8]. A singledeterminant wave function constructed from the KohnSham orbitals [26] shows formation of coreless vortices in much analogy to the ED results in Fig. 2b. Figure 4 shows the separation of the two components with a sharp phase boundary, analogously to the bosonic case. For states with high angular momentum, vorticity becomes large and the DFT solutions show interwoven vor- 
Bosons

a)

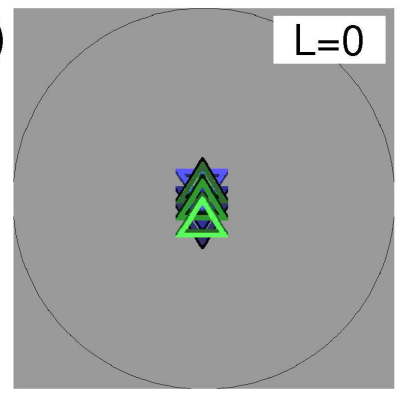

b)

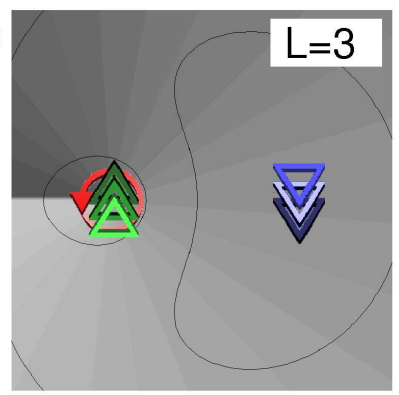

c)

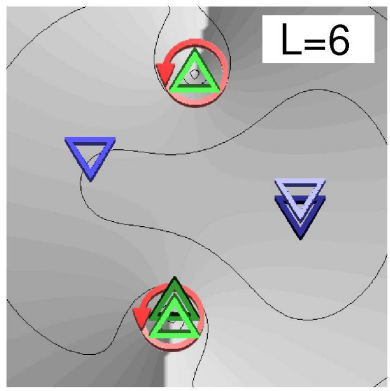

d)

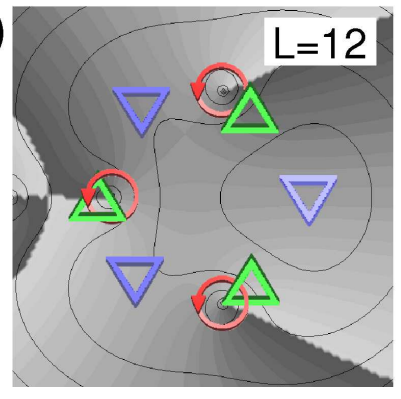

Fermions
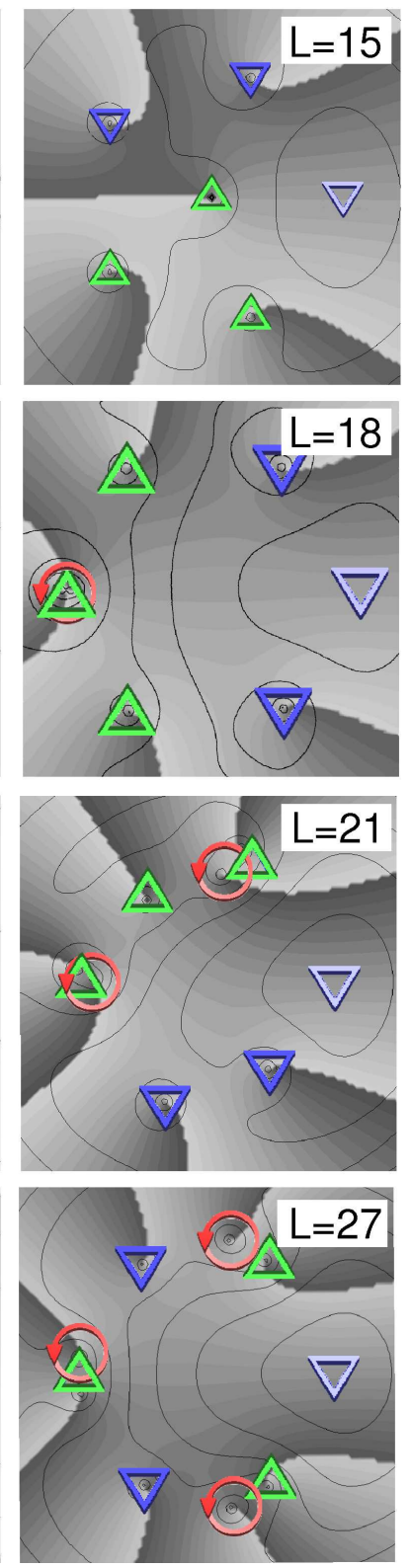

FIG. 2: (Color online): The restricted wave functions calculated with the exact diagonalization method for a two- component system with $N_{\mathrm{A}}=N_{\mathrm{B}}=3$ for bosons at angular momenta $L_{\mathrm{B}}$ (left) and fermionic systems at angular momenta $L_{\mathrm{F}}=L_{\mathrm{B}}+L_{\mathrm{MDD}}=L_{\mathrm{B}}+15$ (right). The states shown are ground states in the lowest Landau level approximation. The bosonic and fermionic states are analogous in nodal structure when one node is removed from each fermion (these nodes are not marked in the right panel for clarity). The figure shows the attachment of b) one, c) two, and d) three vortices, respectively, to particles of the opposite species which indicates the formation of coreless vortices. The notation is explained in the caption of Fig. [1]
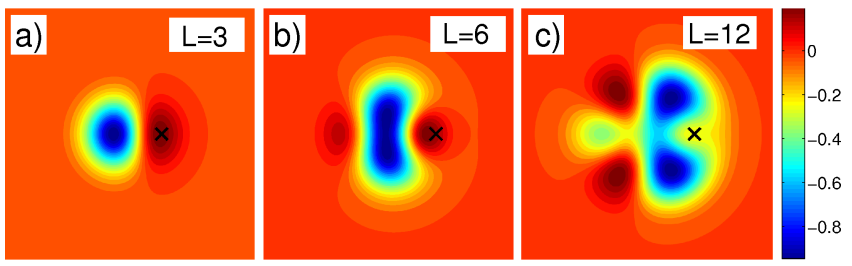

FIG. 3: (Color online): Difference of pair-correlation functions for two species of bosons, for $N_{\mathrm{A}}=N_{\mathrm{B}}=3$. a) Components are separated due to formation of the first coreless vortex. b) Another domain forms due to formation of the second coreless vortex. c) Formation of the third coreless vortex is associated here with "antiferromagnetic" ordering as seen in Fig. 2 $\mathrm{d}$. The reduced wave functions of corresponding states with similar symmetries are shown in Fig. 2b-d.
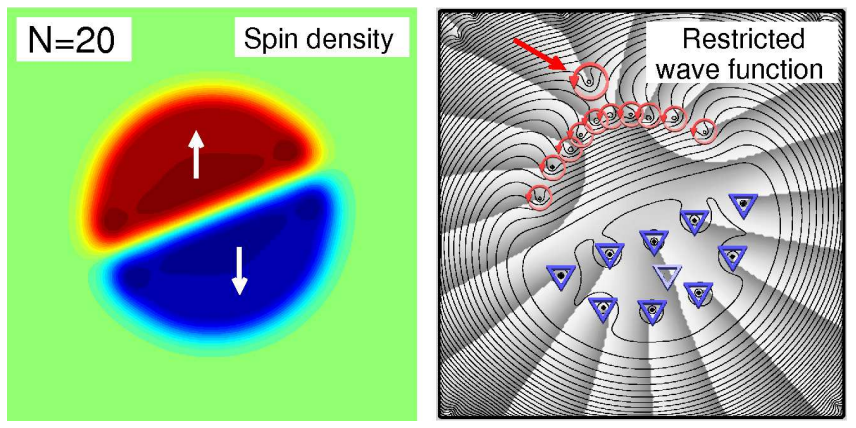

FIG. 4: (Color online): The DFT spin-density (left) in a 20electron droplet with equal number of spins $\left(N_{\uparrow}=N_{\downarrow}=10\right)$ shows separation of spin components into domains. The angular momentum $L=193$ is slightly above the MDD angular momentum $L_{\mathrm{MDD}}=190$. The restricted wave function for the spin-down electrons (right) shows a nodal structure with nodes equal to the number of fixed particles and an additional coreless vortex entering the droplet (arrow).

tex sheets as previously reported for bosonic systems using the GP equations [27].

In the regime where the number of vortices in a bosonic system exceeds the number of particles, the system shows a mixture of coreless vortices and particle-vortex composites. Particularly, the bosonic ground state at $L=21$ shows composites of particles with two vortices in the component of the probing particle, and coreless vortices in the other component, as seen in Fig. 5 a.

The structure of the many-body states analyzed here bear much resemblance with the trial wave functions of non-polarized quantum Hall states. Quantum Hall states are usually polarized in high magnetic fields due to the Zeeman-coupling but some quantum Hall plateaus, such as $\nu=\frac{2}{3}$ and $\nu=\frac{2}{5}$, have been proposed to be nonpolarized states $[28,29]$. The Halperin wave function for such states reads [30]

$$
\psi=\Pi_{i<j}^{N / 2}\left(z_{i}-z_{j}\right)^{q} \Pi_{k<l}^{N / 2}\left(\tilde{z}_{k}-\tilde{z}_{l}\right)^{q} \Pi_{m, n}^{N / 2}\left(z_{m}-\tilde{z}_{n}\right)^{p},
$$

where $q$ is an odd integer (due to fermion antisymmetry) and $p$ is a positive integer and the Gaussians have been 


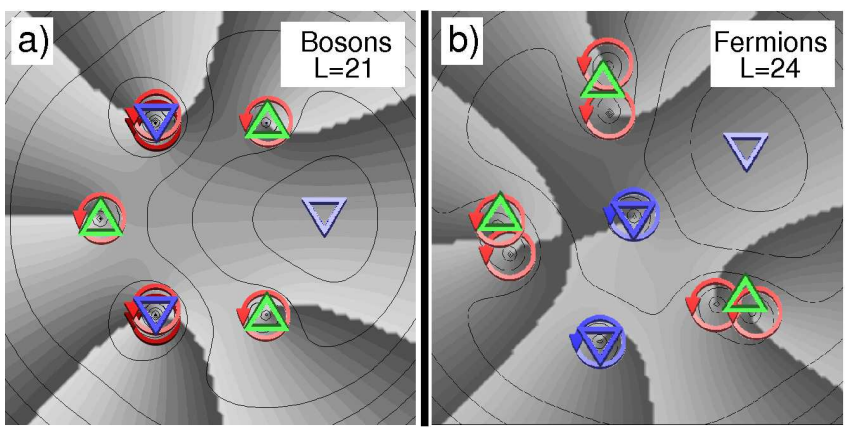

FIG. 5: (Color online): a) In the regime of rapid rotation the bosonic ground state at $L=21$ shows a mixture of both coreless vortices and particle-double-vortex composites b) $L=24$ fermion state with a nodal structure which corresponds to that of the Halperin-wave function with $p=2, q=1$, which attaches two vortices on top of electrons of the opposite spin and one (Pauli) vortex on top of electrons of the same spin. Note that in contrast to Fig. 2 we here mark all nodes.

omitted. This wave function attaches different number of vortices on top of spin up and spin down electrons. A vortex attached to the opposite spin component can be interpreted as a coreless vortex. We find that this nodal structure has a finite-size analogue in two-component fermion droplets. In Ref. [22] the $L=24$ state at $N=6$ was interpreted as a finite-size counterpart of the $\nu=\frac{2}{3}$ quantum Hall state with a Halperin wave function $p=2, q=1$. Figure 5b shows the RWF of this state where one (Pauli) vortex is attached to each particle of the same spin and two (coreless) vortices are attached to particles of the opposite spin, in good agreement with the Halperin model. However, the overlap of this state with the Halperin wave function has been found to be low for large particle numbers due to mixing of spin states in the Halperin wave function (Ref. [22]).

We finally note that even though this study focuses on systems with equal component sizes, coreless vortices also form with unequal component populations. In bosonic systems these have been studied extensively 6]. As a fermionic example we consider results in Ref. 31] where the MDD breakup was analyzed in quantum dots and some beyond-MDD states were found to have partial spin polarization. On the basis of the results presented above, these states can be re-interpreted as vortex states in the majority spin component whose vortex cores are filled with an electron of the minority spin component. Spinselective electron transport experiments could be able to indirectly probe for these types of coreless vortex states in quantum dots [32].

This work was financially supported by the Swedish Research Council, the Swedish Foundation for Strategic Research, and NordForsk.
[1] A. Fetter, e-print arXiv:0801.2952 (2008).

[2] D. A. Butts and D. S. Rokhsar, Nature 397, 327 (1999).

[3] G. M. Kavoulakis, B. Mottelson, and C. J. Pethick, Phys. Rev. A 62, 063605 (2000).

[4] M. R. Matthews, B. P. Anderson, P. C. Haljan, D. S. Hall, C. E. Wieman and E. A. Cornell, Phys. Rev. Lett. 83, 2498 (1999).

[5] J. E. Williams and M. J. Holland, Nature (London) 401, 568 (1999).

[6] K. Kasamatsu, M. Tsubota, and M. Ueda, Int. J. Mod. Phys. B 19, 1835 (2005).

[7] V. Schweikhard, I. Coddington, P. Engels, S. Tung and E.A. Cornell, Phys. Rev. Lett. 93, 210403 (2004).

[8] H. Saarikoski, A. Harju, M. J. Puska, and R. M. Nieminen, Phys. Rev. Lett. 93, 116802 (2004).

[9] M. B. Tavernier and E. Anisimovas and F. M. Peeters, Phys. Rev. B 70, 155321 (2004).

[10] M. Toreblad and M. Borgh and M. Koskinen and M. Manninen and S. M. Reimann, Phys. Rev. Lett. 93, 090407 (2004).

[11] M. Manninen, S. M. Reimann, M. Koskinen, Y. Yu, and M. Toreblad, Phys. Rev. Lett. 94, 106405 (2005).

[12] M. Borgh, M. Koskinen, J. Christensson, M. Manninen, and S. M. Reimann, Phys. Rev. A 77, 033615 (2008).

[13] S. Viefers, J. Phys.: Condens. Matter, 20, 123202 (2008)

[14] K. W. Madison, F. Chevy, W. Wohlleben, and J. Dalibard, Phys. Rev. Lett. 84, 806 (2000).

[15] J. H. Oaknin, L. Martín-Moreno, J. J. Palacios, and C. Tejedor, Phys. Rev. Lett. 74, 5120 (1995).

[16] A. Petković and M. V. Milovanović, Phys. Rev. Lett. 98, 066808 (2007).

[17] N. Yang, J.-L. Zhu, Z. Dai, Y. Wang, cond-mat/0701766

[18] M. A. Baranov, Physics Reports 464, 71 (2008).

[19] S. M. Reimann and M. Manninen, Rev. Mod. Phys. 74, 1283 (2002).

[20] G. Salis, Y. Kato, K. Ensslin, D. C. Driscoll, A. C. Gossard, and D. D. Awschalom, Nature 414, 619 (2001).

[21] C. Weisbuch and C. Hermann, Phys. Rev. B 15, 816 (1977).

[22] M. Koskinen, S. M. Reimann, J.-P. Nikkarila, and M. Manninen, J. Phys. Cond. Matt. 19, 076211 (2007).

[23] A. H. MacDonald, S.-R. Eric Yang, and M. D. Johnson, Aust. J. Phys. 46, 345 (1993).

[24] X. C. Xie, Song He, and S. Das Sarma, Phys. Rev. Lett. 66, 389 (1991).

[25] S. Bargi, J. Christensson, G. M. Kavoulakis, and S. M. Reimann, Phys. Rev. Lett. 98, 130403 (2007).

[26] H. Saarikoski, A. Harju, M. P. Puska, and R. M. Nieminen, Physica E 26, 317 (2005).

[27] K. Kasamatsu, M. Tsubota, and M. Ueda, Phys. Rev. Lett. 91, 150406 (2003).

[28] T. Chakraborty and F. C. Zhang, Phys. Rev. 29, 7032 (1984)

[29] X. C. Xie, Yin Guo, and F. C. Zhang, Phys. Rev. B 40, 3487 (1989).

[30] B. I. Halperin, Helv. Phys. Acta 56, 75 (1983).

[31] S. Siljamäki, A. Harju, R. M. Nieminen, V. A. Sverdlov, and P. Hyvönen, Phys. Rev. B 65, 121306(R) (2002).

[32] K. Hitachi, M. Yamamoto, and S. Tarucha, Phys. Rev. B 74, 161301(R) (2006)

* Electronic address: reimann@matfys.lth.se 